Disponível em

http://www.anpad.org.br/rac

RAC, Curitiba, v. 15, n. 4, art. 1,

pp. 561-579, Jul./Ago. 2011

$\left(\right.$ (c) EYNAC $^{-N}$

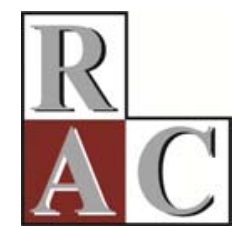

\title{
Extensão de Marcas Corporativas de Serviços: o Efeito da Similaridade Percebida da Extensão e da Qualidade Percebida da Marca
}

\section{Extension of Corporate Services Brands: the Effect of Perceived Similarity Extension and Perceived Quality Brand}

José Mauro da Costa Hernandez *

E-mail: jmhernandez@fei.edu.br Centro Universitário FEI São Paulo, SP, Brasil.

Tassia de Moraes Menck

E-mail: tassia.menck@gmail.com Universidade de São Paulo - EACH/USP São Paulo, SP, Brasil.

Diego Martins G. Lopez E-mail: waxe235@hotmail.com Universidade de São Paulo - EACH/USP

São Paulo, SP, Brasil.

Luiz Fernando T. Mocellin E-mail: luiz.mocellin@itau-unibanco.com.br Universidade de São Paulo - EACH/USP São Paulo, SP, Brasil.

* Endereço: José Mauro da Costa Hernandez

Rua Raul Pompéia, 905, apto. 154, São Paulo/SP, 05025-010.

Copyright (C) 2011 RAC. Todos os direitos, até mesmo de tradução, são reservados. É permitido citar parte de artigos sem autorização prévia, desde que seja identificada a fonte. 


\title{
Resumo
}

O principal objetivo deste estudo é avaliar o efeito da qualidade percebida da marca-mãe e da similaridade percebida sobre as avaliações de extensões de marcas corporativas de serviços. Adicionalmente, também se deseja verificar se a elaboração das características da extensão contribuem para a sua avaliação. As hipóteses foram testadas por meio de três experimentos que envolveram no total 1.131 respondentes. No Estudo 1 foram utilizadas marcas fictícias como estímulo e verificou-se que a qualidade da marca-mãe teve efeito mais importante que a similaridade percebida sobre as avaliações de uma extensão de marca de serviços. No Estudo 2, utilizando como estímulos marcas reais, os mesmos resultados do Estudo 1 foram obtidos. No Estudo 3 verificou-se que a elaboração das características da extensão contribuiu positivamente apenas para uma das extensões propostas para a marca-mãe de alta qualidade, mas não teve nenhum efeito para as extensões propostas para a marca-mãe de baixa qualidade. Tomados em conjunto, os resultados sugerem que a qualidade percebida da marca-mãe tem papel fundamental na avaliação de extensões de marcas corporativas de serviços. O estudo contribui ainda com diversas hipóteses para estudos futuros e implicações gerenciais para gerentes de marcas corporativas de serviços.

Palavras-chave: extensão de marcas; serviços; qualidade da marca; similaridade percebida.

\begin{abstract}
The main objective of this study is to assess the effect of parent brand perceived quality and perceived similarity on the evaluation of corporate service brand extensions. It is also the intention of this study to verify whether providing information cues about the characteristics of the extension contributes to the brand extension evaluation. The hypotheses were tested by means of three experiments involving 1,131 subjects. The results of Study 1, conducted with fictitious brands as stimuli, demonstrated that the perceived quality of the parent brand played a more significant role than perceived similarity on the evaluations of brand extensions. In Study 2, where real brands were used as stimuli, the results were the same as in Study 1. Study 3 found that providing information cues about the characteristics of the extensions had a positive effect for one of the extensions of the high quality parent brand but not for the two proposed extensions of the low quality parent brand. Taken as a whole, the results suggest that the perceived quality of the parent-brand plays a fundamental role in the evaluation of corporate service brand extensions. The study also contributes several hypotheses for future studies and managerial implications for managers of corporate service brands.
\end{abstract}

Key words: brand extensions; services; brand quality; perceived similarity. 


\section{Introdução}

Uma empresa de serviços que deseja entrar em novos mercados pode utilizar o mesmo nome corporativo para nomear as novas ofertas, criar nomes distintos ou combinar estas duas alternativas. À primeira estratégia dá-se o nome de extensão de marcas, isto é, utilizar uma marca existente para lançar um produto ou serviço em uma nova categoria (Aaker \& Keller, 1990). Custos crescentes de mídia, consumidores mais sensíveis a preço e mercados cada vez mais fragmentados tornaram o lançamento de novos produtos e serviços uma atividade muito mais arriscada e, como resultado, a estratégia de extensão de marcas se tornou alternativa muito atraente.

Extensões de marcas promovem lembrança imediata, proporcionando uma forma relativamente fácil e barata para entrar em novos mercados (Grime, Diamantopoulos, \& Smith, 2002). Além de facilitar a aceitação do novo produto, a utilização de marca conhecida para o lançamento de produtos e serviços também tem efeitos benéficos para a marca-mãe: maior eficiência dos investimentos em propaganda (Smith \& Park, 1992), melhoria da imagem da marca (Balachander \& Ghose, 2003) e o aumento da visibilidade da marca-mãe (Swaminathan, Fox, \& Reddy, 2001). Entretanto estender uma marca também oferece riscos, como a diluição das associações centrais da marca (Loken \& John, 1993) e a canibalização das ofertas existentes, caso os dois serviços sejam muito próximos.

A extensão de marcas corporativas de serviços é relativamente comum para nomear serviços similares à atividade principal da marca-mãe, como é costume no caso das empresas financeiras: banco, corretora, seguradora, etc. Entretanto a utilização de uma marca corporativa para o lançamento de categorias de serviços menos similares tem sido menos comum, embora já comecem a surgir alguns exemplos. É o caso das redes de hipermercados Carrefour e Extra que hoje estampam suas marcas em redes de drogarias e postos de gasolina. Por outro lado, marcas internacionais como Disney, Virgin e Microsoft já emprestam seus nomes para uma vasta gama de produtos e serviços há muitos anos. Entretanto a extensão de marcas ainda é muito mais usada por marcas de produtos tangíveis.

A extensão de marcas corporativas de serviços é especialmente atraente porque, dada a complexidade e intangibilidade dos serviços (Iacobucci, 1992), a aquisição de serviços é percebida como mais arriscada que a de um produto tangível (Iacobucci \& Strom, 1996); e a utilização de uma imagem corporativa bem estabelecida pode aumentar a credibilidade e facilitar a percepção de qualidade do novo serviço (Rao, Qu, \& Ruekert, 1999). Uma imagem corporativa também pode ser utilizada para aumentar a eficiência da comunicação, especialmente se o novo serviço está além do escopo atual de atuação da organização (Keller \& Aaker, 1998).

Devido ao uso crescente desta estratégia a partir dos anos 80, diversos teóricos se debruçaram sobre as extensões de marcas, mas a maior parte dos estudos focalizou o estudo de produtos tangíveis e em marcas individuais (Ruyter \& Wetzels, 2000). A despeito da importância do setor de serviços, que no Brasil já representa quase 70\% do PIB (Instituto Brasileiro de Geografia e Estatística [IBGE], 2011), são poucos os estudos que se concentraram em extensões de marcas corporativas de serviços (Pina, Martinez, De Chernatony, \& Drury, 2006).

Investigar extensões de marcas de serviços também se justifica pelo fato de que existem razões teóricas para acreditar que pode haver diferenças entre a capacidade de estender uma marca corporativa de serviços e uma marca de produtos tangíveis. Por exemplo, comparadas com as marcas de produtos, cujas associações são mais específicas e relacionadas ao produto físico, as marcas corporativas possuem associações de natureza mais abstrata (Keller \& Aaker, 1998), o que pode tornar a transferência destas associações mais difícil em determinadas circunstâncias. As características específicas dos serviços - inseparabilidade, heterogeneidade, intangibilidade e perecibilidade - que as diferenciam de produtos tangíveis (Iacobucci, 1998), também sugerem que existam diferenças na avaliação de extensões de marcas de um tipo e de outro (Riel, Lemmink, \& Ouwersloot, 2001).

Já se reconheceu que os principais determinantes do sucesso de extensões de marcas são a similaridade percebida entre a marca-mãe e a nova extensão e a atitude do consumidor em relação à 
marca-mãe (Bottomley \& Holden, 2001; Völckner \& Sattler, 2006), geralmente operacionalizada por meio da qualidade percebida da marca-mãe. Estudos anteriores das extensões de marcas de serviços, entretanto, relataram resultados conflitantes sobre o papel destas duas variáveis. Por exemplo, enquanto o efeito da similaridade percebida foi altamente significativo em alguns estudos das extensões de marcas de serviços (Lei, Pruppers, Ouwersloot, \& Lemmink, 2004; Riel \& Ouwersloot, 2005; Riel et al., 2001; Ruyter \& Wetzels, 2000), Völckner, Sattler, Hennig-Thurau e Ringle (2010) recentemente argumentaram que a qualidade percebida da marca-mãe é o fator de sucesso mais importante e que a similaridade percebida tem efeito muito pequeno. Por outro lado, Pina, Martinez, De Chernatony e Drury (2006) descobriram um efeito muito pequeno da qualidade percebida da marca-mãe.

Os resultados contrastantes de estudos anteriores provavelmente se devem ao uso de diferentes medidas, amostras e desenhos de pesquisa. Independentemente disto, parece evidente que mais estudos são necessários, antes que se possa concluir com mais segurança quais os fatores de sucesso das extensões de marcas de serviços.

Além da importância da qualidade percebida da marca e da similaridade percebida, nenhum estudo anterior de extensões de marcas de serviços examinou conjuntamente o papel destas duas variáveis. Esta é, portanto, a primeira contribuição deste artigo.

Nossa segunda contribuição é avaliar o efeito da elaboração dos atributos da extensão sobre a avaliação da extensão de marca de serviços. Estudos anteriores (Aaker \& Keller, 1990; Boush, 1993; Klink \& Smith, 2001) descobriram que a elaboração dos atributos pode melhorar a avaliação de uma extensão de marcas de produtos tangíveis e nós testamos esta hipótese no contexto de serviços. Finalmente, de forma exploratória, investigamos a influência do grau de intensidade do serviço (Iacobucci, 1998; Shostack, 1977) sobre a avaliação das extensões de serviços.

Especificamente, no primeiro experimento propomos quatro extensões para duas marcas corporativas fictícias de um serviço mais intensivo (hotel) e verificamos a influência da qualidade percebida da marca corporativa e da similaridade percebida. No segundo experimento, aumentamos a validade externa e a generalização dos resultados, ao replicar o primeiro experimento, substituindo o estímulo principal por duas marcas corporativas verdadeiras de um serviço menos intensivo (posto de gasolina). Finalmente, no terceiro experimento, verificamos o efeito da elaboração sobre das características da extensão sobre a avaliação das extensões de duas marcas corporativas de serviços reais associadas a um serviço menos intensivo (supermercado).

A seguir, inicialmente discutimos o papel da similaridade percebida e da qualidade percebida da marca-mãe sobre a avaliação de extensões. Depois, descrevemos os 3 experimentos realizados para testar as hipóteses e concluímos o estudo com sugestões de estudos futuros.

\section{O Papel da Similaridade Percebida na Avaliação de Extensões de Marcas}

Na literatura de extensões de marcas, dois termos são usados indistintamente para indicar o grau em que a extensão de marca e a marca-mãe são semelhantes: adequação (do inglês fit) e similaridade (do inglês similarity). Para Tauber (1988, p. 28), adequação é "quando o consumidor aceita o novo produto como lógico e esperaria que a marca já o tivesse lançado”. Já para Aaker e Keller (1990), adequação é quando um consumidor percebe que o novo item é consistente com a marca-mãe. De modo geral, similaridade ou adequação referem-se ao grau em que a extensão e a marca são parecidas em termos de características, atributos e benefícios (Boush \& Loken, 1991; Park, Milberg, \& Lawson, 1991).

Segundo a teoria da categorização, perspectiva teórica mais aceita para explicar o processo de transferência das associações da marca-mãe para a extensão, o conhecimento dos consumidores sobre produtos e marcas é formado por estruturas parcialmente integradas na memória que, por sua vez, são 
compostas por dois elementos: os objetos percebidos como similares (categoria) e o conhecimento associado a estes objetos. São estas estruturas organizadas de conhecimento que permitem aos indivíduos identificar novos exemplares (por exemplo, uma extensão de marca), classificá-los corretamente em uma das categorias existentes (por exemplo, uma marca) e fazer inferências sobre suas características e julgamentos de avaliação (Boush et al., 1987).

Desta forma, quando o consumidor percebe que um novo produto de uma marca que ele conhece se vem adequando de alguma forma ao seu conhecimento prévio daquela marca, o processo de transferência das associações da marca para a extensão é facilitado (Aaker \& Keller, 1990). Se a similaridade percebida é baixa, além de dificultar a transferência das crenças e atitudes da marca-mãe para o novo produto, o novo produto pode também estimular a formação de associações indesejáveis (Aaker \& Keller, 1990).

Diversos estudos realizados com produtos tangíveis mostraram o seguinte: quanto maior a similaridade percebida entre a extensão e a marca-mãe, melhor a avaliação da extensão da marca (Bottomley \& Holden, 2001; Boush \& Loken, 1991; Keller \& Aaker, 1992; Völckner \& Sattler, 2006). Da mesma forma, a similaridade já se mostrou um importante determinante do sucesso de novas extensões de marcas corporativas de serviços (Lei et al., 2004; Ruyter \& Wetzels, 2000). Portanto, com base na discussão acima e nos resultados de estudos anteriores, formulamos a seguinte hipótese.

H1: Quanto maior a similaridade percebida entre a marca corporativa de serviços e a extensão, melhor a avaliação da extensão.

\section{O Papel da Qualidade Percebida da Marca Corporativa de Serviços na Avaliação de Extensões de Marcas}

Qualidade pode ser definida como a avaliação global dos consumidores sobre a superioridade ou excelência de um produto ou serviço (Zeithaml, 1988). Esta definição sugere que a qualidade percebida possui um nível superior de abstração ao que os atributos específicos relacionados ao produto ou serviço representam.

Parece claro supor que uma marca-mãe que não tem associações positivas como, por exemplo, qualidade superior, não pode transferir às suas extensões as associações que as favoreçam imediatamente. Entretanto resultados anteriores mostraram que a qualidade da marca, por si só, também não é capaz de garantir o sucesso de uma extensão. Para Aaker e Keller (1990), quando a similaridade percebida é baixa, o processo de transferência das associações da marca-mãe para a extensão é inibido. Nesta condição, é mais provável que a avaliação da extensão seja feita apenas com base nas crenças inferidas no momento do julgamento. A similaridade percebida funciona como catalisador que facilita a transferência das associações da marca-mãe para a extensão (Boush \& Loken, 1991).

Estudos anteriores mostraram que há uma interação da qualidade da marca-mãe com a similaridade da extensão (Aaker \& Keller, 1990; Bottomley \& Holden, 2001; Wölckner \& Sattler, 2006). Este mesmo efeito de interação também foi observado em estudos que envolvem marcas de serviços (Pina et al., 2006; Riel et al., 2001); portanto, formulamos a seguinte hipótese.

H2: Para marcas corporativas de serviços de alta qualidade percebida, as extensões de alta similaridade percebida, serão mais bem avaliadas que as extensões de baixa similaridade percebida, enquanto extensões de marcas corporativas de serviços de baixa qualidade percebida serão mal avaliadas, independentemente da similaridade percebida.

A seguir descrevemos o primeiro experimento realizado para testar estas duas hipóteses. 


\section{Estudo 1}

O Estudo 1 foi desenhado para testar as duas hipóteses no contexto de uma marca fictícia. Optamos por uma marca fictícia, para evitar efeitos de confusão decorrentes do conhecimento ou do afeto em relação à marca, além dos efeitos manipulados pelo experimento (Keller, 1993). Também optamos por utilizar neste estudo uma categoria de serviço mais intensivo (hotel), isto é, categoria em que os serviços têm papel mais preponderante sobre a oferta do que os produtos tangíveis (Shostack, 1977). As extensões propostas também se caracterizam por serem mais intensivas em serviços e foram escolhidas de forma a variarem em função do nível de similaridade percebida. Para a escolha das categorias das extensões a serem propostas, realizamos um pré-teste, descrito a seguir.

\section{Seleção dos estímulos}

Estudantes universitários de grande universidade brasileira ( $\mathrm{n}=59$ ), com idade média de 21 anos, avaliaram 22 categorias de serviços em termos de similaridade com hotéis. Utilizamos as mesmas medidas de similaridade utilizadas por Aaker e Keller (1990), isto é, complementaridade, substituibilidade e transferibilidade. Os sujeitos foram apresentados às definições de cada conceito e solicitados a avaliar, em escala de 7 pontos (1=nada ou quase nada; $7=$ totalmente ou quase totalmente), quanto cada categoria proposta era complementar, substituta ou transferível em relação a um hotel. As definições foram feitas da seguinte forma:

"Produtos complementares são aqueles que podem ser utilizados em conjunto e ao mesmo tempo (por exemplo, sorvete e cobertura).

Produtos substitutos são aqueles que o consumidor pode comprar um em lugar do outro (por exemplo, suco e refrigerante).

Produtos transferíveis são aqueles para os quais uma mesma empresa tem pessoas, instalações e habilidades para fazer os dois produtos ou serviços: por exemplo, isqueiro e caneta podem ser feitos facilmente pela mesma empresa, porque utilizam o plástico como matéria-prima."

Uma medida de similaridade global foi calculada, a partir da média dos valores destas 3 medidas e os serviços de restaurante $(M=5,1)$ e escola de idiomas $(M=2,5 ; t(58)=14,1, p<0,1 \%)$ emergiram como bons candidatos para o estudo principal.

Neste mesmo pré-teste, também avaliamos a descrição de duas marcas fictícias de redes de hotéis, para verificar se elas eram percebidas de forma diferente em relação à qualidade. As descrições proporcionadas aos respondentes foram as seguintes (entre parêntesis, as frases que foram modificadas para compor o estímulo alternativo):

'Imagine que a rede de hotéis $\mathbf{X}$ é bem conhecida e o nível de serviços prestados sempre recebeu boas avaliações (tem recebido avaliações ruins) de seus clientes e das revistas especializadas em turismo. Provavelmente você já foi uma ou mais vezes a um dos hotéis da rede da marca $\mathbf{X}$ e ficou muito bem impressionado (desapontado) com o bom atendimento (atendimento ruim) e o ambiente aconchegante (pouco aconchegante). Imagine que a sua impressão sobre a rede de hotéis $\mathbf{X}$ é muito favorável (desfavorável).’

Pedimos aos 59 sujeitos lerem uma destas descrições e avaliarem a qualidade do hotel descrito, numa escala de 7 pontos: 1=péssima qualidade; 7=excelente qualidade. Como esperado, a rede de hotéis descrita como possuindo alta qualidade $(\mathrm{M}=5,9)$ teve média superior de qualidade percebida à rede de hotéis descrita como possuindo baixa qualidade ( $\mathrm{M}=3,2 ; \mathrm{t}(57)=7,9 ; \mathrm{p}<0,1 \%)$. 


\section{Método}

Experimentos baseados em cenários têm sido muito utilizados em estudos de extensões de marcas (Boush \& Loken, 1991; Fedorikin et al., 2008). Especificamente, neste primeiro experimento utilizamos um desenho fatorial completo entre sujeitos 2 (similaridade percebida: alta vs. baixa) X 2 (qualidade da marca corporativa de serviços: alta vs. baixa). Oito mil pessoas de um banco de dados particular receberam um convite por e-mail para participarem de uma pesquisa postada no site questionpro.com. Um novo convite foi enviado uma semana após o envio da primeira mensagem. Por se tratar de um experimento entre sujeitos, cada elemento da amostra recebeu o convite para responder a apenas um dos questionários. A coleta de dados foi encerrada 15 dias após o envio da segunda mensagem, quando havia 302 respondentes (3,8\% de taxa de resposta).

Em termos de características demográficas, a amostra final ficou bem dividida entre os dois sexos (49\% de homens). A idade média dos respondentes foi de 42 anos e, em geral, os respondentes tinham alto nível de escolaridade - 30\% com curso superior completo e 51\% com pós-graduação.

\section{Procedimentos e medidas}

Inicialmente, pedimos aos respondentes que lessem a descrição de uma rede de hotéis e avaliassem a sua qualidade, utilizando escala de 7 pontos: 1=péssima qualidade; 7=excelente qualidade. A seguir, pedimos a eles que imaginassem que a rede de hotéis estava pretendendo lançar novo serviço, descrito de forma a proporcionar informações suficientes para que o respondente pudesse compreender que se tratava de extensão e assim poder avaliar o novo serviço. No texto da proposta da rede de restaurantes, podia-se ler o seguinte:

'A rede de hotéis $\mathbf{X}$ pretende lançar um novo serviço: uma rede de restaurantes. Nos restaurantes da rede $\mathbf{X}$ você poderá desfrutar de diversos pratos da cozinha internacional, em ambiente adequado para este tipo de cozinha. Localizadas nas principais capitais do país, todas as unidades da rede de restaurantes $\mathbf{X}$ contam com os serviços comuns aos restaurantes do seu porte.'

Já para a extensão de uma rede de escola de idiomas, os respondentes foram solicitados a ler o seguinte texto:

'A rede de hotéis $\mathbf{X}$ pretende lançar novo serviço: uma rede de escola de idiomas. As escolas de idiomas $\mathbf{X}$ oferecem cursos de inglês, espanhol e francês para adultos, jovens e crianças em diversos horários. Suas unidades, distribuídas por todo o país, contam com laboratórios de informática e multimídia e bibliotecas.’

A avaliação das extensões foi operacionalizada de forma similar àquela utilizada por Aaker e Keller (1990). Os respondentes foram solicitados a avaliar a qualidade da extensão ('Eu acho que ... terá alta qualidade') utilizando uma escala de Likert de 7 pontos (1=discordo totalmente; 7=concordo totalmente) e a intenção de utilizar o serviço, avaliada por uma escala de diferencial semântico de 7 pontos: 1=extremamente improvável; 7=extremamente provável. A correlação entre as duas medidas foi de $76 \%(\mathrm{p}<1 \%)$, proporcionando um bom nível de consistência interna. Assim, as duas medidas foram agregadas para compor a atitude em relação às extensões propostas.

Os respondentes avaliaram o grau de similaridade percebida entre hotéis e as extensões propostas, utilizando as mesmas escalas do pré-teste (complementariedade, substituibilidade, transferibilidade) e mais uma medida adicional de similaridade global (Alfa de Crombach=0,73). A medida de similaridade foi calculada a partir da média dos 4 indicadores.

\section{Resultados}

Para checar a manipulação das variáveis, rodamos duas ANOVA 2 (similaridade percebida: alta vs. baixa) X 2 (qualidade percebida da marca-mãe: alta vs. baixa) sobre as medidas de similaridade 
percebida e qualidade percebida da marca-mãe. Como esperávamos, para a variável dependente similaridade percebida, observamos apenas um efeito principal da similaridade percebida $(\mathrm{F}(1,298)=124,7, \mathrm{p}<0,1 \%)$ e nenhum outro efeito. Por outro lado, para a variável dependente qualidade percebida da marca-mãe, observamos apenas um efeito principal da qualidade percebida $(F(1,298)=1079,7, p<0,1 \%)$ e nenhum outro efeito.

Para testar a Hipótese 1, também rodamos uma ANOVA 2 (similaridade percebida: alta vs. baixa) X 2 (qualidade percebida da marca: alta vs. baixa) sobre a variável dependente avaliação da extensão. Observamos um efeito principal da qualidade percebida $(F(1,298)=30,54, p<0,1 \%)$, um efeito principal da similaridade percebida $(F(1,298)=158,88, \mathrm{p}<0,1 \%)$ e um efeito significativo de interação $(F(1,298)=10,06, p<1 \%)$. Desta forma, não podemos rejeitar nem H1 nem H2.

Análises post-hoc mostraram que para a marca-mãe de baixa qualidade percebida, a avaliação da extensão de alta similaridade percebida $(M=3,1)$ não foi significativamente diferente da avaliação da extensão de baixa similaridade percebida $(M=2,7)$; $t(158)=1,6, p>10 \%)$. Entretanto, para a marcamãe de alta qualidade percebida, a avaliação da extensão de alta similaridade percebida $(M=5,5)$ foi significantemente maior que a avaliação da extensão de baixa similaridade percebida $(M=4,2$; $\mathrm{t}(140)=6,4, \mathrm{p}<0,1 \%)$. O gráfico a seguir ilustra os resultados das avaliações de cada extensão obtidas no Estudo 1.

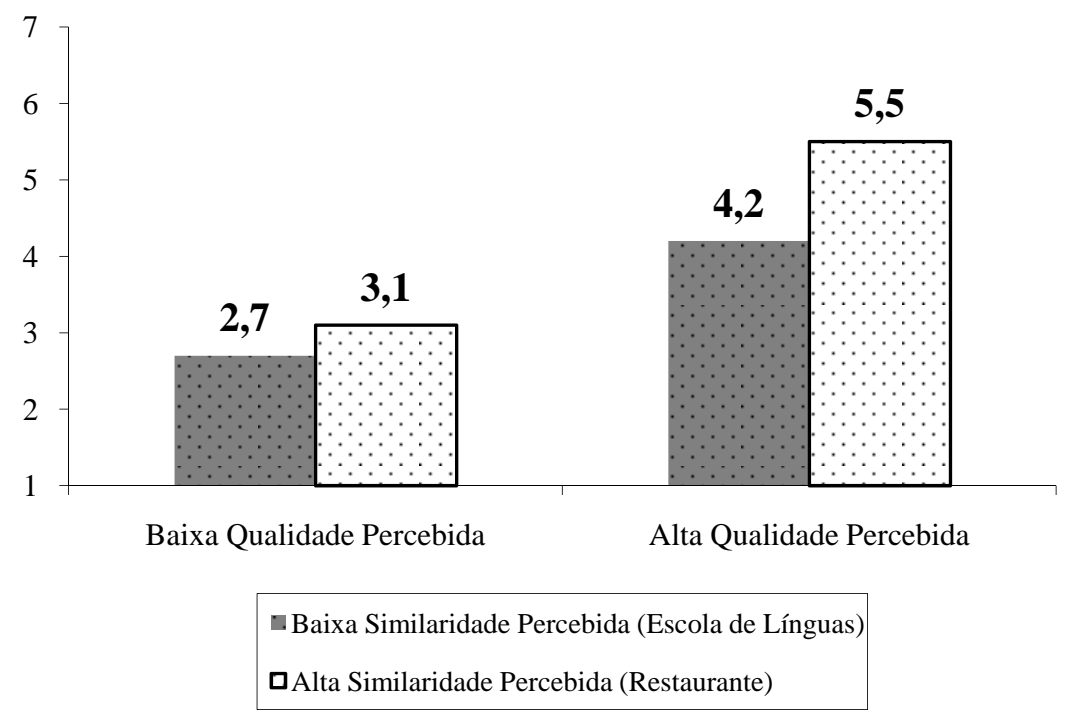

Figura 1. Avaliações das Extensões Propostas do Estudo 1.

\section{Discussão}

No Estudo 1 verificamos que a qualidade percebida da marca-mãe teve papel mais preponderante que a similaridade percebida sobre a avaliação de extensões de marcas de serviços, já que a similaridade percebida não teve nenhum efeito, quando a qualidade da marca-mãe era baixa. Por outro lado, a extensão de maior similaridade percebida foi mais bem avaliada, quando a qualidade percebida da marca-mãe era alta.

Embora não sejam diretamente comparáveis com os resultados de estudos anteriores de extensões de marcas de serviços, dadas as diferenças metodológicas, os resultados do Estudo 1 estão em linha com aqueles obtidos por Riel e Ouwersloot (2005) e Riel, Lemmink e Ouwersloot (2001), porquanto eles encontraram um efeito de interação entre qualidade percebida e algumas dimensões de similaridade percebida. Entretanto, os resultados são diferentes daqueles encontrados por Pina et al. (2006) e Völckner et al. (2010), visto que eles não observaram este mesmo efeito de interação.

Os resultados do Estudo 1 são similares àqueles verificados no estudo de Keller e Aaker (1992), no contexto de produtos tangíveis e, assim como eles, concluímos que marcas de serviços de alta 
qualidade têm maior capacidade de extensibilidade que marcas de baixa qualidade. Este resultado é importante na literatura de serviços, porque esclarece evidências contraditórias obtidas em estudos anteriores sobre o papel da similaridade percebida e da qualidade percebida da marca-mãe sobre a avaliação de extensões.

Apesar dos resultados satisfatórios do Estudo 1, a utilização de marcas fictícias ameaça a validade externa do experimento. Para eliminar esta limitação, no Estudo 2 replicamos o Estudo 1, utilizando marcas reais como estímulo.

\section{Estudo 2}

O objetivo do Estudo 2 é replicar os efeitos obtidos no Estudo 1, em contexto de marcas reais e em categoria de serviços diferente. De modo geral, utilizamos os mesmos procedimentos e medidas utilizadas no Estudo 1 e, para facilidade da leitura, descrevemos em detalhes apenas as diferenças entre cada estudo.

Selecionamos como categoria de serviços para o estímulo da marca-mãe uma rede de postos de gasolina, por se tratar de serviço menos intensivo, devido à comercialização de produtos tangíveis: gasolina e derivados. Por termos utilizado marcas reais, ao invés de marcas fictícias, incluímos na análise duas covariáveis: familiaridade com a marca (Broniarczyk \& Alba, 1994) e apego emocional (Fedorikhin, Park, \& Thomsom, 2008). Optamos por apego emocional ao invés de outras medidas de afeto, porque Fedorikhin, Park e Thomsom (2008) mostraram que apego emocional está além das dimensões afetivas de atitude e desempenha papel independente na avaliação de extensões de marcas. Logo, o uso de apego emocional proporciona um teste mais estrito para as nossas hipóteses. Tanto as marcas de alta e baixa qualidade quanto as categorias de serviços das extensões propostas como estímulos foram obtidas a partir de 2 pré-testes, descritos a seguir.

\section{Seleção dos estímulos}

Submetemos 8 marcas (Ale, BR Petrobras, Via Brasil, Esso, Ipiranga, Repsol, Shell e Texaco) à avaliação de 40 estudantes de graduação (idade média $=21$ ). Solicitamos a eles que indicassem numa escala de 7 pontos (1=péssima qualidade; $7=$ excelente qualidade) a qualidade de cada uma das redes de postos de gasolina que eles conhecessem.

A partir dos resultados desta coleta de dados, selecionamos as marcas BR Petrobras $(M=5,2)$ e Via Brasil $(\mathrm{M}=2,5 ; \mathrm{t}(24)=6,2 ; \mathrm{p}<1 \%)$. Embora a rede BR Petrobras fosse conhecida por todos os respondentes, a rede de postos Via Brasil era conhecida por apenas 63\% dos respondentes.

Em seguida, solicitamos a outros 30 alunos de graduação (idade média $=21$ ) que indicassem 0 grau de similaridade percebida entre postos de gasolina e 17 tipos de serviços. Com base nestas respostas, selecionamos para estímulo uma rede de lojas de serviços automotivos $(M=4,8)$ e uma rede de livrarias $(\mathrm{M}=3,1 ; \mathrm{t}(29)=8,8 ; \mathrm{p}<0,01)$.

\section{Método}

Utilizamos um desenho fatorial completo entre sujeitos 2 (similaridade percebida: alta vs. baixa) X 2 (qualidade percebida da marca-mãe: alta vs. baixa). Foram enviados 2 convites por e-mail para 8 mil pessoas que não haviam participado do Estudo 1, com intervalo de uma semana; a coleta de dados se encerrou 15 dias após o segundo envio. A amostra final foi composta por 250 respondentes (3,2\% de taxa de resposta), com ligeira preponderância de pessoas do sexo masculino (54\%). A idade média foi 35 anos e, como no Estudo 1, os respondentes tinham alto nível de escolaridade - 27\% tinha curso superior completo e $44 \%$ tinha pós-graduação. 


\section{Procedimentos e medidas}

Inicialmente, os respondentes foram solicitados a indicar a qualidade das marcas de postos de gasolina e o grau de familiaridade com cada marca, numa escala de diferencial semântico de 7 pontos (1=não conheço nada; 7=conheço muito bem). Em seguida, cada respondente avaliou o grau de apego emocional em relação à marca, utilizando a mesma escala de Fedorikhin et al. (2008). Os itens da escala foram traduzidos para o português e, na sua forma final, ficaram da seguinte maneira: 'Eu me sinto ligado(a) emocionalmente à ...', 'A marca ... é apaixonante', 'Eu sou leal à ...', 'A marca ... é cativante’ e 'A marca ... é encantadora’. O Alfa de Crombach para os 5 itens foi igual a 0,94 e a medida consolidada foi obtida a partir da média dos 5 indicadores.

A seguir, cada respondente leu um texto que lhes solicitava imaginar que a marca que eles estavam avaliando pretendia lançar um novo serviço. O texto para a rede de lojas de serviços automotivos era o seguinte:

'A rede de postos Via Brasil (BR Petrobras) pretende lançar um novo serviço: uma rede de lojas de serviços automotivos. Nesta nova rede de lojas de serviços automotivos Via Brasil (BR Petrobras) você poderá trocar os pneus, amortecedores, freios, suspensão e escapamento do seu veículo, além de poder contar com vários outros serviços como balanceamento, alinhamento e troca de óleo. As lojas de serviços automotivos Via Brasil (BR Petrobras) também estarão preparadas para fazer uma revisão completa no seu carro.'

Já o texto para a rede de livrarias podia ser lido da seguinte forma:

'A rede de postos Via Brasil (BR Petrobras) pretende lançar um novo serviço: uma rede de livrarias. Nas livrarias da rede Via Brasil (BR Petrobras) você terá acesso a um vasto acervo literário, incluindo livros acadêmicos, infantis e de ficção, além de dicionários. Nas livrarias da Via Brasil (BR Petrobras) você também encontrará uma papelaria e um grande acervo de revistas.'

A correlação entre a qualidade percebida da extensão e a intenção de frequentar a extensão foi igual a $74 \%(\mathrm{p}<0,01)$ e as duas medidas foram consolidadas em uma única variável. Em seguida, os respondentes avaliaram o grau de similaridade percebida entre postos de gasolina e as extensões propostas, utilizando os mesmos procedimentos já descritos no Estudo 1.

\section{Resultados}

Para checar a manipulação das variáveis, rodamos duas ANOVA 2 (similaridade percebida: alta vs. baixa) X 2 (qualidade percebida da marca-mãe: alta vs. baixa) sobre as medidas de similaridade percebida e qualidade percebida da marca-mãe. Como esperávamos, para a variável dependente similaridade percebida, observamos apenas um efeito principal da similaridade percebida $(\mathrm{F}(1,246)=76,87, \mathrm{p}<0.1 \%)$ e nenhum outro efeito. Por outro lado, para a variável dependente qualidade percebida da marca-mãe, observamos apenas um efeito principal da qualidade percebida $(F(1,246)=157,4, p<0,1 \%)$ e nenhum outro efeito.

Para testar as Hipóteses 1 e 2, rodamos uma ANOVA (similaridade percebida: alta vs. baixa) X 2 (qualidade percebida da marca: alta vs. baixa) sobre a variável dependente avaliação da extensão e com as covariáveis mencionadas: familiaridade com a marca-mãe e apego emocional. Observamos um efeito principal da qualidade percebida $(\mathrm{F}(1,244)=5,9, \mathrm{p}<5 \%)$, um efeito principal da similaridade percebida $(F(1,244)=3,0, p<, 10 \%)$ e um efeito significativo de interação $(F(1,244)=3,2, p<10 \%)$. Estes resultados não nos permitem rejeitar nem $\mathrm{H} 1$ nem $\mathrm{H} 2$. Também verificamos forte efeito de apego emocional $(\mathrm{F}(1,244)=56,9, \quad \mathrm{p}<0,1 \%)$, mas nenhum efeito de familiaridade com a marca-mãe $(\mathrm{F}(1,244)=2,1, \mathrm{p}>10 \%)$.

Análises post-hoc mostraram que para a marca-mãe de baixa qualidade percebida, a avaliação da extensão de alta similaridade percebida $(M=3,1)$ não foi significativamente diferente da avaliação da extensão de baixa similaridade percebida $(M=3,1)$; $t(113)=0,9, p>10 \%)$. Entretanto, para a marca- 
mãe de alta qualidade percebida, a avaliação da extensão de alta similaridade percebida $(M=4,4)$ foi significativamente maior que a avaliação da extensão de baixa similaridade percebida $(\mathrm{M}=4,0$; $\mathrm{t}(133)=1,7, \mathrm{p}<10 \%)$. O gráfico a seguir ilustra os resultados das avaliações de cada extensão obtidas no Estudo 2.

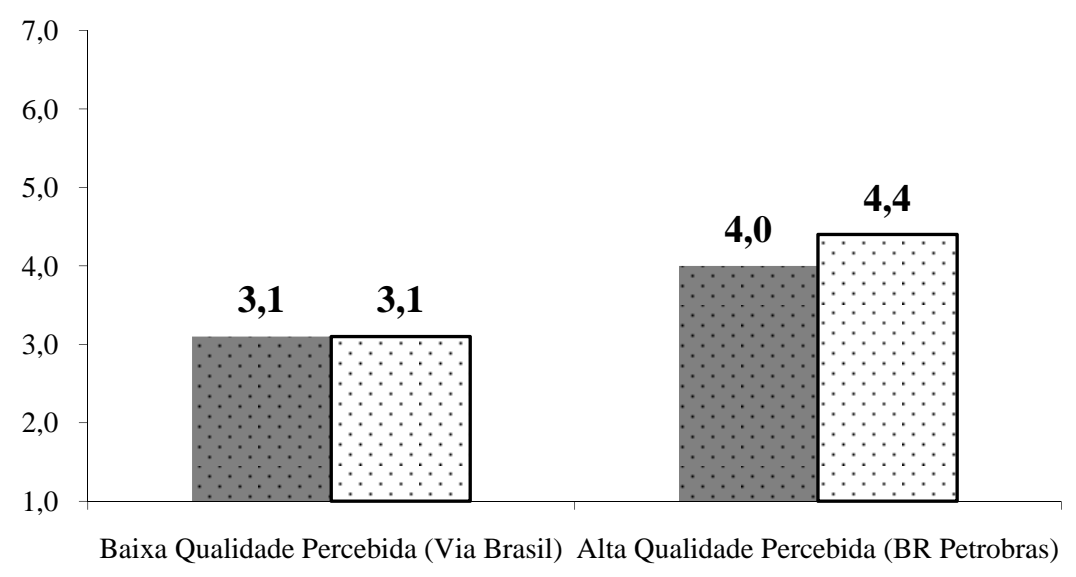

• Baixa Similaridade (Livraria) ロAlta Similaridade (Loja de Serviços Automotivos)

Figura 2. Avaliações das Extensões Propostas do Estudo 2.

\section{Discussão}

No Estudo 2, encontramos os mesmos resultados que já havíamos encontrado no Estudo 1, isto é, não observamos diferenças nas avaliações das extensões propostas para uma marca-mãe de baixa qualidade percebida, mas verificamos que a similaridade percebida teve efeito positivo para as avaliações das extensões propostas da marca-mãe de alta qualidade percebida.

O Estudo 2 proporciona evidências ainda mais fortes que o Estudo 1, no sentido de que a qualidade percebida da marca-mãe exerce papel mais importante que a similaridade percebida sobre a avaliação de extensões de marcas. No Estudo 1, pode-se argumentar que a importância da qualidade percebida foi mais saliente, dada a manipulação da qualidade percebida das marcas fictícias, resultando uma diferença maior entre a qualidade percebida das marcas (Ms=5,7 vs. 2,0, respectivamente, para as marcas-mãe de alta qualidade e baixa qualidade) e que as extensões avaliadas eram mais similares em relação à categoria da marca-mãe (Ms=4,1 vs. 2,6, respectivamente, para as extensões propostas de maior similaridade e menor similaridade). Entretanto este argumento não é válido no Estudo 2, já que a diferença de qualidade percebida diminuiu sensivelmente em relação ao Estudo 1 (Ms=5,4 vs. 3,3, respectivamente, para as marcas-mãe de alta qualidade e baixa qualidade), enquanto as extensões propostas eram menos similares em relação à categoria da marca-mãe $(\mathrm{Ms}=3,1$ vs. 2,1, respectivamente, para as extensões propostas de maior similaridade e menor similaridade).

Além disso, o Estudo 2 aumenta a validade do Estudo 1 de duas formas: primeiro, ao utilizarmos marcas reais, aumentamos a validade externa; segundo, ao invés de usar um serviço mais intensivo (hotel), usamos um serviço menos intensivo (posto de gasolina).

Outro ponto interessante a observar é que o efeito do apego emocional afeto em relação à marca foi bastante significativo. Estes resultados estão em linha com aqueles observados por Fedorikhin et al. (2008) e Yeung e Wyer (2005), sugerindo que, em determinadas circunstâncias, o apego emocional em relação à marca pode ser mais preponderante que a própria similaridade percebida. Já o conhecimento da marca não teve nenhum efeito, levando a supor que a utilização de marcas reais não ameaçou a validade do experimento. 


\section{Estudo 3}

Em situações de compras reais, os consumidores costumam ter mais informações sobre as extensões do que apenas a marca e a categoria de serviços (Aaker \& Keller, 1990; Klink \& Smith, 2001). A propaganda de uma nova extensão de marca pode aumentar a saliência das associações da marca que ajudam o consumidor a fazer inferências sobre as características e os benefícios da extensão e, portanto, compreender a possível similaridade existente entre a marca-mãe e a extensão (Völckner \& Sattler, 2006). Estudos anteriores das extensões de marcas mostraram que as suas avaliações podem modificar-se ao se proporcionar mais informações dos atributos dos produtos estendidos (Aaker \& Keller, 1990; Boush, 1993; Klink \& Smith, 2001). Entretanto, Aaker e Keller (1990) descobriram que proporcionar informações dos atributos de qualidade da marca-mãe não funcionou tão eficientemente quanto proporcionar informações adicionais dos atributos da extensão. A elaboração dos atributos da extensão tornou a similaridade entre as categorias mais saliente, contribuindo para a avaliação da extensão.

Também já se demonstrou que pode haver diferenças individuais que dificultam a percepção da similaridade entre a marca-mãe e a nova extensão. Por exemplo, Kim e John (2008) mostraram que indivíduos que interpretam seu ambiente em níveis superiores, em termos de características abstratas ou generalizadas, atribuem mais importância à similaridade percebida do que indivíduos que interpretam seu ambiente em níveis inferiores, em termos de características concretas e contextualizadas. Para estes últimos, se a similaridade não for tornada mais clara, a dificuldade em estabelecer uma relação entre a marca-mãe e a extensão é maior, contribuindo para a pior avaliação da extensão.

Entretanto, assim como foi argumentado anteriormente, a elaboração das características da extensão pode não funcionar, quando a marca é de baixa qualidade, já que não há atitudes positivas a serem transferidas para a nova extensão. Da mesma forma, é mais provável que esta elaboração funcione melhor para uma extensão de média similaridade percebida, já que uma extensão de baixa (alta) similaridade percebida tenderá a ser menos bem (mais bem) avaliada, tornando mais difícil verificar o efeito da elaboração das características da extensão numa condição experimental. Logo, a seguinte hipótese pode ser formulada.

H3: Para marcas-mãe de alta qualidade, a avaliação de uma nova extensão de marca de média similaridade percebida é melhor, quando se proporcionam informações sobre os atributos da nova oferta do que quando não se proporcionam estas informações, mas esta diferença não se verifica para marcas-mãe de baixa qualidade.

No Estudo 3, a hipótese acima foi testada em contexto de marcas reais e utilizando, como estímulo, diferentes categorias para as extensões (serviços mais e menos intensivos) de marca associada com categoria de serviço menos intensivo (supermercados), isto é, além da marca, também manipulamos a intensidade de serviços da extensão de marca e a elaboração dos atributos da extensão.

\section{Seleção dos estímulos}

Pedimos a 31 estudantes de graduação que indicassem a qualidade percebida de 16 marcas de supermercados e, com base nos resultados, selecionamos as marcas Pão de Açúcar $(M=6,5)$ e Dia\% $(\mathrm{M}=4,0 ; \mathrm{t}(27)=9,8 ; \mathrm{p}<0,1 \%)$ para o experimento.

Também solicitamos a eles que indicassem a similaridade percebida entre supermercados e 29 categorias de serviços, escolhidas propositadamente para variarem em termos de intensidade de serviços. A similaridade percebida variou entre 1,3 (escritório de advocacia) e 5,2 (lanchonete), numa escala de 7 pontos. Com base nestes resultados, escolhemos duas categorias de serviços que possuíam média similaridade percebida em relação a supermercados. A primeira, mais intensiva em serviços, foi 
o serviço de entregas rápidas $(M=3,6)$ e a segunda, menos intensiva em serviços, foi uma rede de livrarias $(\mathrm{M}=3,8 ; \mathrm{t}(30)=0,8 ; \mathrm{p}>10 \%$.).

\section{Método}

Utilizamos um desenho fatorial completo entre sujeitos 2 (qualidade percebida da marca-mãe: alta vs. baixa) X 2 (intensidade do serviço: mais intensivo vs. menos intensivo) X 2 (elaboração dos atributos da extensão: com vs. sem). Enviamos 15 mil convites para as pessoas responderem a um questionário postado na Internet (questionpro.com). Após uma semana, novo convite foi enviado e a coleta de dados se encerrou 15 dias após o envio deste último convite.

A amostra final foi composta por 572 respondentes (3,8\% de taxa de resposta), tendo ficado bem dividida entre os dois sexos (48\% de homens). A idade média dos respondentes foi de 35 anos e com alto grau de escolaridade - $27 \%$ tinha curso superior completo e $54 \%$ tinha pós-graduação.

\section{Procedimentos e medidas}

Inicialmente os respondentes foram solicitados a indicar a qualidade, a familiaridade e o apego emocional em relação às marcas-mãe de supermercado, utilizando as mesmas escalas do Estudo 2. Em seguida, cada respondente foi solicitado a imaginar que a marca que eles estavam avaliando pretendia lançar um novo serviço. Para a extensão de entregas rápidas, um dos textos elaborou sobre o conhecimento de logística da rede de supermercados, a qualidade do atendimento nas lojas e os locais em que as entregas poderiam ser feitas. Os textos com e sem a elaboração das características da extensão de entregas rápidas apresentados aos respondentes foram os seguintes (em negrito, a elaboração sobre as características da extensão):

'A rede de supermercados Pão de Açúcar (Dia\%) pretende lançar um novo serviço: Entregas Rápidas Pão de Açúcar (Dia\%). (Utilizando todo o seu conhecimento em logística, o Pão de Açúcar (Dia\%) pretende proporcionar um serviço com a mesma qualidade que você já está acostumado a encontrar em suas lojas. As remessas poderão ser feitas em qualquer uma das várias lojas da rede presentes em diversas cidades brasileiras). Com este novo serviço, você poderá enviar documentos e objetos para qualquer lugar do mundo de forma rápida e confiável.'

Para a extensão de livrarias, um dos textos elaborou sobre a experiência em negociação das redes de supermercados, a qualidade do atendimento e a proximidade das lojas de cada rede. Os textos para a extensão de livrarias com e sem elaboração podiam ser lidos da seguinte forma (em itálico, a elaboração sobre as características da extensão):

'A rede de supermercados Pão de Açúcar (Dia\%) pretende lançar um novo serviço: Livrarias Pão de Açúcar (Dia\%). (Utilizando a sua vasta experiência em negociação com fornecedores e no varejo de produtos de consumo, o Pão de Açúcar (Dia\%) pretende oferecer um serviço com a mesma qualidade que você já está acostumado a encontrar em suas lojas. Assim como os supermercados da rede Pão de Açúcar (Dia\%), você encontrará uma livraria Pão de Açúcar (Dia\%) sempre perto de você). Com este novo serviço, você terá acesso a uma grande variedade de títulos de livros, CDs, DVDs, games, softwares e revistas.'

A atitude em relação às novas extensões foi operacionalizada por meio de uma medida de qualidade da extensão e intenção de utilizar o serviço $\left(\mathrm{r}^{2}=0,59 ; \mathrm{p}<1 \%\right)$. Os respondentes avaliaram o grau de similaridade percebida entre supermercados e as extensões propostas, utilizando as mesmas medidas dos outros dois estudos.

\section{Resultados}

Para checar a manipulação, rodamos duas ANOVA 2 (qualidade da marca-mãe: alta vs. baixa) X 2 (intensidade de serviço da extensão: alta vs. baixa) X 2 (elaboração de atributos: com vs. sem). A 
primeira, utilizando como variável dependente qualidade percebida da marca-mãe, revelou apenas um efeito principal da qualidade da marca-mãe $(F(1,564)=331,0, p<0,1 \%)$ e nenhum outro efeito. A segunda, utilizando como variável dependente a similaridade percebida não revelou nenhum efeito, significando que as duas extensões tinham a mesma similaridade percebida; portanto concluímos que tanto a manipulação da qualidade da marca-mãe quanto da similaridade funcionaram.

Para testar a Hipótese 3, rodamos uma ANOVA 2 (qualidade da marca-mãe: alta vs. baixa) X 2 (intensidade de serviço da extensão: alta vs. baixa) X 2 (elaboração de atributos: com vs. sem), incluindo as duas covariáveis mencionadas (familiaridade com a marca-mãe e apego emocional em relação a marca-mãe) e como variável dependente a avaliação da extensão de marca.

Os resultados mostraram um efeito principal da marca-mãe $(F(1,562)=32,3, p<0,1 \%)$ e um efeito principal da elaboração de atributos $(\mathrm{F}(1,562)=10,2, \mathrm{p}<1 \%)$. Ao contrário do que havíamos previsto em H2, não verificamos efeito de interação da marca com a elaboração. Entretanto mais interessante foi verificar um efeito de interação da marca com a elaboração e intensidade de serviços $(F(1,562)=5,0, p<5 \%)$. Também verificamos forte efeito do apego emocional $(F(1,562)=90,1, p<0,1 \%)$ e efeito da familiaridade $(\mathrm{F}(1,562)=8,1, \mathrm{p}<1 \%)$.

A seguir, investigamos mais de perto a natureza da interação das 3 variáveis. Análises post-hoc mostraram que, para o serviço menos intensivo (livraria), a elaboração dos atributos não melhorou a avaliação das extensões propostas. Especificamente, para a marca de baixa qualidade percebida (Dia\%), não houve diferenças entre as avaliações das extensões com e sem elaboração (Ms=2,9 vs. $3,0, \mathrm{t}(139)=0,3, \mathrm{p}>10 \%)$; e o mesmo resultado foi observado para as extensões da marca de alta qualidade percebida (Pão de Açúcar), isto é, não houve diferença entre as avaliações das extensões com e sem elaboração (Ms=3,7 vs. 3,7, $\mathrm{t}(134)=0,4, \mathrm{p}>10 \%$ ). Por outro lado, para o serviço mais intensivo (entregas rápidas), a elaboração dos atributos melhorou a avaliação da extensão da marca de alta qualidade (Pão de Açúcar) (Ms=3,4 vs. 4,1, t(145)=4,5, p<0,1\%); mas não melhorou a avaliação da extensão da marca de baixa qualidade (Dia\%) (Ms=2,7 vs. 2,9, t(146)=1,3, p>10\%). A Figura 3 resume as avaliações das extensões propostas no Estudo 3.
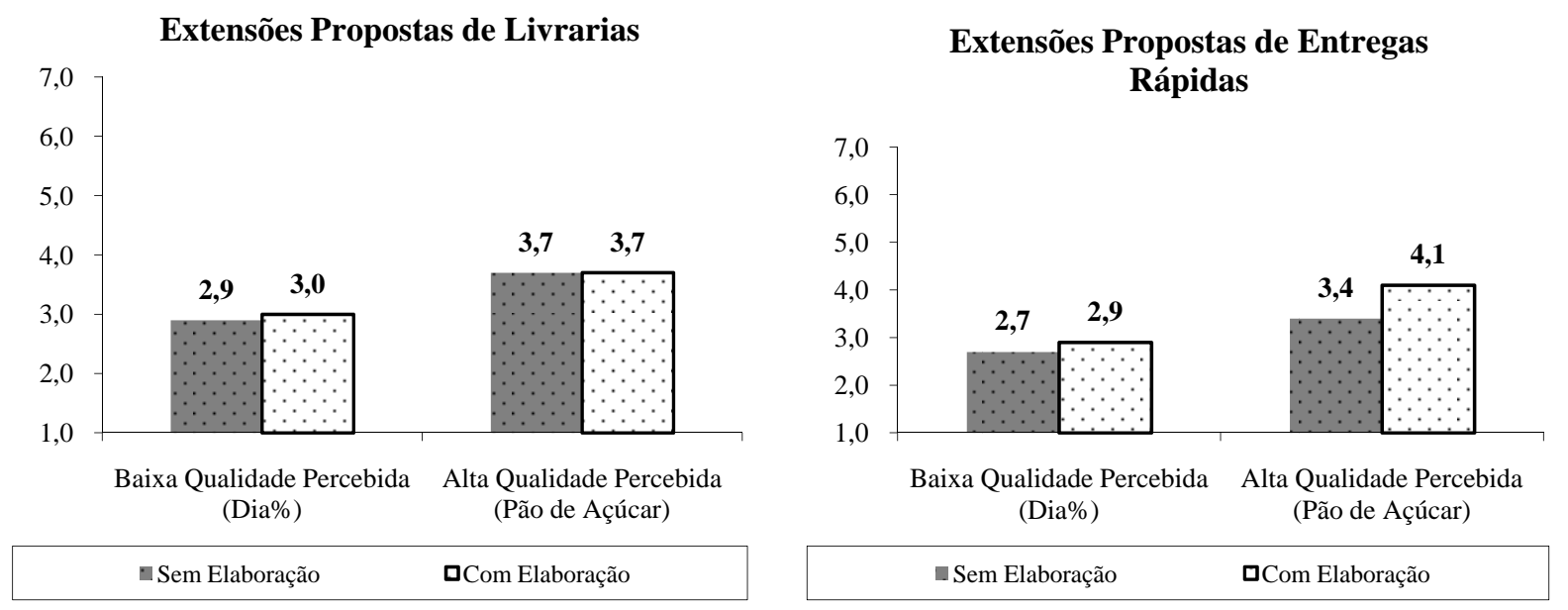

Figura 3. Avaliações das Extensões Propostas do Estudo 3.

Para compreender melhor o papel da elaboração dos atributos da extensão, isolamos as extensões da marca-mãe de alta qualidade e comparamos as avaliações. Percebemos que, quando não houve elaboração, a extensão para o serviço menos intensivo foi mais bem avaliada que a extensão para o serviço mais intensivo ( $\mathrm{Ms}=3,7$ vs. $3,4, \mathrm{t}(130)=1,9, \mathrm{p}<10 \%$ ) ao passo que, quando houve elaboração, a extensão para o serviço menos intensivo foi mais bem avaliada que a extensão para o serviço mais intensivo (Ms=3,7 vs. 4,1, $\mathrm{t}(149)=3,1, \mathrm{p}<1 \%$ ). Se lembrarmos que os supermercados representam uma categoria menos intensiva de serviços, ainda que de forma exploratória, estes resultados sugerem uma relação interessante: na ausência de elaboração, uma extensão de serviço 
menos intensivo é mais bem avaliada quando a marca-mãe está associada a um serviço menos intensivo, ou seja, por terem graus similares de intensividade, a extensão seria mais facilmente compreendida como extensão mais lógica.

Quando a categoria da extensão não tem o mesmo grau de intensidade da categoria da marcamãe, o consumidor não estabelece uma relação imediata entre os dois e faz uma avaliação menos positiva da extensão. Na presença da elaboração, o consumidor compreende mais facilmente a relação entre as duas categorias e melhora a sua avaliação. Isto explicaria por que a extensão mais intensiva (entregas rápidas) foi mais bem avaliada que a categoria de serviços menos intensiva (livraria) na presença da elaboração.

\section{Discussão}

No Estudo 3, verificamos novamente o importante papel que a qualidade percebida da marcamãe desempenha na avaliação de suas extensões de marcas. Na condição da marca-mãe de baixa qualidade percebida, a elaboração dos atributos da extensão não teve nenhum efeito sobre a avaliação das extensões. Resultados similares foram encontrados nos Estudos 1 e 2, isto é, marcas-mãe de baixa qualidade têm capacidade reduzida de se estenderem.

Embora não tenhamos observado o efeito de interação da qualidade percebida da marca-mãe com a elaboração dos atributos da extensão, observamos um resultado mais interessante, já que o efeito da elaboração foi diferente para os dois serviços (mais e menos intensivos). Para o serviço menos intensivo (livraria), a elaboração dos atributos da extensão não teve nenhum efeito sobre as avaliações. Já para o serviço mais intensivo (entregas rápidas), a elaboração melhorou a avaliação das extensões propostas para a marca-mãe de alta qualidade percebida, mas não a avaliação das extensões propostas da marca-mãe de baixa qualidade percebida.

Uma explicação plausível para este padrão de resultados é que os sujeitos perceberam a extensão proposta de livrarias como mais lógica para um supermercado e proporcionar mais informações sobre a extensão não melhorou a avaliação da extensão. Tanto é assim que, sem elaboração, a extensão livraria foi mais bem avaliada que a extensão entregas rápidas para a marca de alta qualidade. Por outro lado, a extensão de entregas rápidas pode ter sido percebida como menos lógica como extensão de um supermercado e as informações adicionais esclareceram que habilidades um supermercado teria, para poder lançar um serviço de entregas rápidas. Neste caso, a extensão entregas rápidas passou a ser mais bem avaliada, até mesmo que a extensão de livrarias.

Estudos sobre impressão de informações (Lichtenstein \& Srull, 1987) podem explicar este fenômeno: quando os sujeitos são expostos a uma informação considerada diagnóstica (um supermercado estendendo-se para uma livraria), ele desconta as informações subsequentes (elaboração dos atributos da extensão) e a primeira unidade de informação fornecida influencia mais o julgamento. Isto explicaria por que a elaboração dos atributos não teve nenhum efeito sobre a avaliação da livraria para a marca-mãe de alta qualidade. Entretanto, quando a primeira unidade de informação não é diagnóstica (um supermercado estendendo-se para entregas rápidas), informações adicionais (elaboração dos atributos) têm maior influência sobre o julgamento do indivíduo de tal forma que os dois conjuntos de informações influenciam conjuntamente o julgamento. Isto explicaria por que a avaliação da extensão entregas rápidas da marca-mãe de alta qualidade melhorou com a elaboração dos atributos.

\section{Conclusão}

Estimulados pela relativa ausência, na literatura de marketing de estudos sobre extensões de marcas de serviços, nosso objetivo com este estudo era avaliar o papel da qualidade da marca-mãe e da similaridade percebida sobre as avaliações de extensões de marcas corporativas de serviços. 
Exploratoriamente, também investigamos diferentes categorias de serviços, variando em grau de intensidade (Shostack, 1977).

Por meio de 3 experimentos, submetemos diversas extensões de marcas corporativas de serviços à avaliação de 1.131 consumidores. Nos dois primeiros experimentos, a similaridade percebida mais alta só melhorou a avaliação das extensões propostas para as marcas-mãe de alta qualidade. No terceiro experimento, a elaboração dos atributos da extensão só melhorou a avaliação da extensão proposta da marca-mãe de alta qualidade percebida, quando a extensão pertencia a um serviço mais intensivo, mas não quando a extensão pertencia a um serviço menos intensivo.

Existem evidências contraditórias em estudos anteriores de extensões de marcas de serviços sobre o papel da qualidade percebida da marca-mãe e da similaridade percebida (Pina et al., 2006; Völckner et al., 2010). Nossos estudos mostram claramente que o papel da qualidade percebida da marca-mãe é significativamente mais importante que a similaridade percebida no contexto de serviços.

Para gerentes de marcas de serviços, os resultados apenas ressaltam a importância dos investimentos na construção e manutenção do brand equity das marcas de serviços. Embora estudos anteriores não tenham encontrado evidências de que a marca seja mais importante para serviços do que é para produtos tangíveis (Krishnan \& Hartline, 2001), os resultados relatados aqui demonstram claramente que marcas de serviços de alta qualidade percebida têm muito mais facilidade de se estenderem do que marcas de baixa qualidade percebida. Neste sentido, os resultados estão muito próximos daqueles encontrados por Keller e Aaker (1992).

Como é costume em experimentos, manipulamos a qualidade percebida da marca-mãe e a similaridade percebida apenas em dois níveis (alto e baixo). Estudos futuros poderiam superar esta limitação, manipulando níveis intermediários para se descobrir qual a sensibilidade da avaliação das extensões de marcas a diferentes níveis de qualidade percebida da marca-mãe e similaridade percebida.

Também verificamos que a elaboração dos atributos da extensão funcionou apenas para a extensão de um serviço mais intensivo (entregas rápidas) de uma marca-mãe de alta qualidade (Pão de Açúcar) associada a um serviço menos intensivo (supermercado). De acordo com a nossa interpretação, a elaboração funcionou apenas porque a extensão de um serviço mais intensivo possa ter sido percebida como menos lógica para uma marca associada a um serviço menos intensivo, levando os respondentes a questionarem as habilidades da marca-mãe em oferecer o novo serviço. Este resultado sugere duas hipóteses que merecem ser estudadas no futuro.

A primeira é que a intensidade de serviços pode indicar as habilidades e capacidades de uma marca corporativa de serviços, bem como as suas fronteiras de extensão. Por exemplo, um varejista de eletrônicos, serviço que pode ser considerado menos intensivo, poderia usar com maior probabilidade de sucesso sua experiência em negociação, logística e pessoal para estender sua marca para outro tipo de varejo (também um serviço menos intensivo) do que, por exemplo, para serviço de TV a cabo (um serviço mais intensivo). Estudos futuros poderiam examinar mais atentamente quais as associações que emergem em diferentes condições de extensões de marcas de serviços para diferentes tipos de intensidade de serviços e como estas associações se relacionam à avaliação da extensão.

A segunda hipótese é que a elaboração dos atributos da extensão funciona apenas quando os consumidores não conseguem imediatamente conectar a marca-mãe à categoria da extensão. Neste caso, é mais provável que os consumidores tentem resolver a inconsistência e estejam mais abertos a novas informações proporcionadas pela propaganda. Por outro lado, se a conexão é prontamente estabelecida, os consumidores não estarão dispostos a prestar atenção em mais informações e a elaboração dos atributos não funcionará. Embora os resultados obtidos aqui sejam apenas de natureza exploratória, parece-nos que esta hipótese também mereça estudos futuros.

Para gerentes de marcas de serviços, a implicação é que a mensagem de uma propaganda de extensões de marcas deve estar adequada às condições de similaridade percebida. Por exemplo, se as extensões são muito similares, talvez valha mais a pena convencer o consumidor das vantagens do 
novo serviço do que tentar convencê-lo de que o novo serviço é legítimo. Entretanto, se as extensões são menos similares, é aconselhável antes tentar convencer o consumidor de que a nova extensão é legítima.

No terceiro experimento, ao isolarmos os resultados das extensões da marca-mãe de alta qualidade percebida, observamos que a extensão de um serviço menos intensivo foi mais bem avaliada que a extensão de um serviço mais intensivo antes da elaboração dos atributos da extensão. Baseados neste resultado, sugerimos que extensões de serviços mais intensivos são mais bem avaliadas, quando estendidas por marcas-mãe associadas a serviços mais intensivos enquanto extensões de serviços menos intensivos são mais bem avaliadas, quando estendidas por marcas-mãe associadas a serviços menos intensivos. Esta hipótese é diferente daquela sugerida por Lei, Pruppers, Ouwersloot e Lemmink (2004), segundo a qual extensões mais intensivas em serviços são mais bem avaliadas do que extensões menos intensivas em serviços. Ainda que baseada em resultados exploratórios, nossa hipótese está baseada no pressuposto de que a intensidade de serviços implica certas habilidades e capacidades de uma marca que podem ser transferidas mais facilmente para outras categorias que compartilhem o mesmo grau de intensidade de serviços. Estudos futuros poderiam testar esta hipótese com desenhos experimentais, em que tanto a intensidade da categoria de serviços da marca-mãe quanto as extensões propostas são manipuladas.

Finalmente, cumpre destacar o importante papel que o apego emocional desempenhou nos 2 estudos em que se utilizaram como estímulos marcas reais. Acreditamos que mais estudos sejam necessários para se identificar o significado que o apego emocional desempenha em extensões de marcas de serviços.

Artigo recebido em 02.07.2010. Aprovado em 09.05.2011.

\section{Referências}

Aaker, D. A., \& Keller, K. L. (1990). Consumer evaluations of brand extensions. Journal of Marketing, 54(1), 27-41. doi: 10.2307/1252171

Balachander, S., \& Ghose, S. (2003). Reciprocal spillover effects: a strategic benefit of brand extensions. Journal of Marketing, 67(1), 4-13. doi: 10.1509/jmkg.67.1.4.18594

Bottomley, P. A., \& Holden, S. J. S. (2001). Do we really know how consumers evaluate brand extensions? Empirical generalizations based on secondary analysis of eight studies. Journal of Marketing Research, 38(4), 494-500. doi: 10.1509/jmkr.38.4.494.18901

Boush, D. M. (1993). How advertising slogans can prime evaluations of brand extensions. Psychology and Marketing, 10(1), 67-78. doi: 10.1002/mar.4220100106

Boush, D. M., \& Loken, B. (1991). A process-tracing study of brand extension evaluation. Journal of Marketing Research, 28(1), 16-28. doi: 10.2307/3172723

Boush, D. M., Shipp, S., Loken, B., Gencturk, E., Crockett, S., Kennedy, E., Bettie, M., Misurell, D., Rochford, L., \& Strobel, J. (1987). Affect generalization to similar and dissimilar brand extensions. Psychology and Marketing, 4(3), 225-237. doi: 10.1002/mar.4220040306

Broniarczyk, S. M., \& Alba, J. W. (1994). The importance of the brand in brand extension. Journal of Marketing Research, 31(2), 214-228. doi:10.2307/3152195.

Fedorikhin, A., Park, C. W., \& Thomson, M. (2008). Beyond fit and attitude: the effect of emotional attachment on consumer responses to brand extensions. Journal of Consumer Psychology, 18(4), 281-291. doi: 10.1016/j.jcps.2008.09.006 
Grime, I., Diamantopoulos, A., \& Smith, G. (2002). Consumer evaluations of extensions and their effects on the core brand. Key issues and research propositions. European Journal of Marketing, 36(11-12), 1415-1438. doi: 10.1108/03090560210445245

Iacobucci, D. (1992). An empirical examination of some basic tenets in services: goods-services continua. In T. A. Swartz, D. E. Bowen, \& S. W. Brwon (Eds.), Advances in services marketing and management: research and practice (pp. 23-52). New York: JAI Press.

Iacobucci, D. (1998). Services: what do we know and where shall we go? A view from marketing. In T. A. Swartz, D. E. Bowen, \& S. W. Brwon (Eds.), Advances in services marketing and management (1-96). New York: JAI Press.

Iacobucci, D., \& Ostrom, A. (1996). Perceptions of services. Journal of Retailing and Consumer Services, 3(4), 195-212. doi: 10.1016/0969-6989(95)00072-0

Instituto Brasileiro de Geografia e Estatística. (2011). Contas nacionais e trimestrais. Indicadores de volume $e$ valores correntes. Recuperado em 21 junho, 2011, de http://www.ibge.gov.br/home/estatistica/indicadores/pib/pib-vol-val_201101caderno.pdf

Keller, K. L. (1993). Conceptualizing, measuring, and managing customer-based brand equity. Journal of Marketing, 57(1), 1-22. doi: 10.2307/1252054

Keller, K. L., \& Aaker, D. A. (1992). The effects of sequential introduction of brand extensions. Journal of Marketing Research, 29(1), 35-50.

Keller, K. L., \& Aaker, D. A. (1998). The impact of corporate marketing on a company's brand extensions. Corporate Reputation Review, 1(4), 356-378. doi: 10.1057/palgrave.crr.1540057

Kim, H., \& John, D. R. (2008). Consumer response to brand extensions: construal level as a moderator of the importance of perceived fit. Journal of Consumer Psychology, 18(2), 116-126. doi: 10.1016/j.jcps.2008.01.006

Klink, R. R., \& Smith, D. C. (2001). Threats to the external validity of brand extension research. Journal of Marketing Research, 37(3), 326-335. doi: 10.1509/jmkr.38.3.326.18864

Krishnan, B. C., \& Hartline, M. D. (2001). Brand equity: is it more important in services? Journal of Services Marketing, 15(5), 328-342. doi: 10.1108/EUM0000000005654

Lei, J., Pruppers, R., Ouwersloot, H., \& Lemmink, J. (2004). Service intensiveness and brand extension evaluations. Journal of Service Research, 6(3), 243-255. doi: $10.1177 / 1094670503259381$

Loken, B., \& John, D. R. (1993). Diluting brand beliefs: when do brand extensions have a negative impact? Journal of Marketing, 57(3), 71-84. doi: 10.2307/1251855

Park, C. W., Milberg, S., \& Lawson, R. (1991). Evaluation of brand extensions: the role of perceived fit and brand concept consistency. Journal of Consumer Research, 18(2), 185-193. doi: $10.1086 / 209251$

Pina, J. M., Martinez, E., Chernatony, L. de, \& Drury, S. (2006). The effect of service brand extensions on corporate image: an empirical model. European Journal of Marketing, 40(1-2), 174-197. doi: 10.1108/03090560610637374

Rao, A. R., Qu, L., \& Ruekert, R. W. (1999). Signaling unobservable product quality through a brand ally. Journal of Marketing Research, 36(2), 258-268. doi: 10.2307/3152097

Riel, A. C. R. van, Lemmink, J., \& Ouwersloot, H. (2001). Consumer evaluations of service brand extensions. Journal of Service Research, 3(3), 220-231. doi: 10.1177/109467050133003 
Riel, A. C. R. van, \& Ouwersloot, H. (2005). Extending electronic portals with new services: exploring the usefulness of brand extension models. Journal of retailing and consumer services, 12(4), 245-254. doi: 10.1016/j.jretconser.2004.07.003

Ruyter, K., \& Wetzels, M. (2000). The role of corporate image and extension similarity in service brand extensions. Journal of Economic Psychology, 21(6), 639-659. doi: 10.1016/S01674870(00)00024-6

Shostack, G. L. (1977). Breaking free from product marketing. The Journal of Marketing, 41(2), 7380. doi: $10.2307 / 1250637$

Smith, D. C., \& Park, C. W. (1992). The effects of brand extensions on market share and advertising efficiency. Journal of Marketing Research, 29(3), 296-313. doi: 10.2307/3172741

Swaminathan, V., Fox, R. J., \& Reddy, S. K. (2001). The impact of brand extension introduction on choice. Journal of Marketing, 65(4), 1-15. doi: 10.1509/jmkg.65.4.1.18388

Tauber, E. M. (1988). Brand leverage: strategy for growth in a cost-controlled world. Journal of Advertising Research, 28(4), 26-30.

Völckner, F., \& Sattler, H. (2006). Drivers of brand extension success. Journal of Marketing, 70(2), 18-34. doi: 10.1509/jmkg.70.2.18

Völckner, F., Sattler, H., Hennig-Thurau, T., \& Ringle, C. M. (2010). The role of parent brand quality for service brand extension success. Journal of Service Research, 13(4), 379-396. doi: $10.1177 / 109467051037054$

Yeung, C. W. M., \& Wyer, R. S., Jr. (2005). Does loving a brand mean loving its products? The role of brand-elicited affect in brand extension evaluations. Journal of Marketing Research, 42(4), 495-506. doi: 10.1509/jmkr.2005.42.4.495

Zeithmal, V. A. (1988). Consumer perceptions of price, quality, and value: a means-end model and synthesis of evidence. Journal of Marketing, 52(3), 2 - 22. doi: 10.2307/1251446 